\title{
Dynamique Demographique, Nutrition Et Alimentation Dans La Commune De Karimama Au Benin
}

\author{
Edoun Emmanuel Guy, \\ Mongbo L. Roch, \\ École Doctorale Pluridisciplinaire (EDP), Laboratoire d'Analyse des \\ Dynamiques Sociales et des Etudes du Développement (LADYD), \\ Université d'Abomey-Calavi (UAC), Bénin
}

Doi:10.19044/esj.2020.v16n27p331 URL:http://dx.doi.org/10.19044/esj.2020.v16n27p331

\section{Résumé}

L'augmentation du nombre de bouches à nourrir engendré par le rythme effréné de la croissance démographique et la réduction des terres disponibles pour l'agriculture limitent les efforts pour assurer la nutrition et la sécurité alimentaire. Dans la Commune de Karimama, la forte pression qui s'exerce sur les terres agricoles a un impact négatif considérable sur la satisfaction des besoins alimentaires des populations. De quelle façon l'accroissement peu maîtrisé de la population affecte-il l'accès à l'alimentaire dans cette Commune ? Afin de répondre à cette question, cette étude se propose de s'intéresser au rythme de la croissance démographique pour mieux comprendre l'accès des ménages vulnérables à l'alimentation et leur résilience à la sécurité alimentaire. Elle a été réalisée à travers une recherche socio anthropologique qui s'appuie sur des données quantitatives et qualitatives. L'analyse a révélé que la Commune de Karimama enregistre un fort taux annuel de croissance démographique $4,72 \%$. Cette croissance rapide augmente la pression sur la terre et conduit à une parcellisation dans la Commune. La superficie agricole par tête des membres du ménage est à peine égale à $0,68 \mathrm{ha} / \mathrm{tête}$. La production alimentaire largement en deçà des besoins des populations se traduit par un proxy calorifique chroniquement inférieur à la norme de 2400 $\mathrm{kcal} / \mathrm{habts} /$ jours. L'insécurité alimentaire touche plus d'une personne sur deux $(54 \%)$. La Commune rencontre également de grandes difficultés pour l'accès de ses populations à la santé ( 1 seul médecin pour plus de 76000 habitants en 2018) et à l'éducation où le taux de scolarisation surtout des jeunes filles est la plus faible de la région de l'Alibori.

Pour une culture de résilience en sécurité alimentaire et nutritionnelle dans la Commune, la maîtrise de la fécondité par l'accès à la fois des femmes 
à la contraception et des jeunes filles à une éducation de qualité n'est pas une option mais une priorité.

Mots clés: Dynamique Démographique, Alimentation, Nutrition, Résilience Et Bénin.

\title{
Demographic Dynamics, Nutrition And Food In The Commune Of Karimama In Benin
}

\author{
Edoun Emmanuel Guy, \\ Mongbo L. Roch, \\ École Doctorale Pluridisciplinaire (EDP), Laboratoire d'Analyse des \\ Dynamiques Sociales et des Etudes du Développement (LADYD), \\ Université d'Abomey-Calavi (UAC), Bénin
}

\begin{abstract}
The increase in the number of mouths to feed caused by the rapid rate of population growth and the reduction in the land available for agriculture are limiting efforts to ensure nutrition and food security. In the Commune of Karimama, the strong pressure exerted on agricultural land has a considerable negative impact on the satisfaction of the food needs of the populations. How does the poorly controlled population growth affect access to food in this Municipality? In order to answer this question, this study aims to look at the rate of population growth in order to better understand vulnerable households' access to food and their resilience to food security. It was carried out through socio-anthropological research that is based on quantitative and qualitative data. The analysis revealed that the Municipality of Karimama has a strong annual population growth rate of $4.72 \%$. This rapid growth increases the pressure on the land and leads to fragmentation in the Commune. The agricultural area per head of household members is barely 0.68 ha / head. Food production far below the needs of the populations results in a calorific proxy chronically lower than the standard of $2400 \mathrm{kcal} /$ habts / day. Food insecurity affects more than one in two people (54\%). The Municipality also encounters great difficulties for the access of its populations to health (only 1 doctor for more than 76,000 inhabitants in 2018) and to education where the enrollment rate, especially for young girls, is the lowest in the Alibori region.

For a culture of resilience in food and nutritional security in the Municipality, controlling fertility through access by both women to contraception and young girls to quality education is not an option but a priority.
\end{abstract}


Keywords: Population Dynamics, Food, Nutrition, Resilience, Benin

\section{Introduction}

L'accroissement rapide de la population et la hausse des besoins alimentaires accroissent la pression qui s'exerce sur les ressources naturelles. La croissance démographique met à mal les efforts déployés pour réduire la pauvreté, créer des emplois et assurer la sécurité alimentaire, énergétique et hydrique, tout en protégeant l'environnement naturel (Forum économique mondial et UNFPA, 2012). Ainsi, pour nourrir une population mondiale de neuf (09) milliards d'individus, chiffre qui sera probablement atteint avant le milieu de ce siècle, il faudra que la production agricole progresse globalement de $70 \%$ environ (FAO, 2010). Et si nous n'agissons pas de toute urgence, le monde pourrait compter 16 milliards d'habitants en 2100 (UNFPA, 2011b ; Nations unies, 2011). Les pays de l'Afrique et notamment ceux de l'Afrique subsaharienne ne sont pas restés en marge de cette croissance démographique. Avec un taux d'accroissement de $2.4 \%$ en 2008 et $2.5 \%$ pour l'Afrique subsaharienne contre $1.2 \%$ pour le monde, l'Afrique est devenue la région d'explosion démographique. En 2007, l'Afrique subsaharienne abritait 807 millions d'habitants, soit $84 \%$ de la population du continent africain; la progression africaine est donc essentiellement liée à celle de l'Afrique subsaharienne (K. A. Djinadou, 2012). La population est un facteur déterminant l'augmentation de la demande en denrées alimentaires. La FAO affirme que d'ici à 2050, le facteur principal de hausse des besoins sera l'accroissement de la population mondiale. Il faudra ainsi compléter les régimes alimentaires afin d'éliminer la sous-alimentation chronique. Le supplément à payer avoisinera $20 \%$ des disponibilités alimentaires pour le monde en développement. Les pays en développement devraient donc accroître leurs disponibilités en énergie d'origine végétale de $175 \%$, l'Afrique de $414 \%$, et les peuples qui s'alimentent surtout de manioc, d'igname, ou de taro, de 617 \% (FAO, 2013). Le Bénin, un pays de l'Afrique subsaharienne, connait aussi une augmentation rapide de sa population. En 2013, le quatrième recensement de la population a dénombré 10008749 habitants contre 6 769914 habitants au recensement de 2002, soit un taux annuel d'accroissement intercensitaire de 3,5\% (INSAE/RGPH4, 2013). La population béninoise a été multipliée par quatre depuis 1960, et elle continue de s'accroître de quelque 300000 personnes par an. La fécondité reste élevée au Bénin, 4,9 enfants/ménage en 2012 et 5,7 en 2014 ((EDSB, 2012 ; MICS, 2014). Cette fécondité est encore plus élevée en milieu rurale comme la commune de Karimama où le taux d'accroissement annuel de la population est de 4,72\%. Cette croissance démographique rapide a pour conséquence une augmentation de bouches à nourrir et la nécessité de produire plus de vivres. C'est une réalité 
que la croissance démographique galopante à Karimama entraîne une forte augmentation des besoins alimentaires et nutritionnels. Dans cette Commune où la population a été multipliée par plus de 4 fois en moins de 40 ans, comment se présente la situation alimentaire et nutritionnelle des ménages ? Avec ses terres très limitées, ses problèmes de conflits pour la terre et l'eau entre agriculteurs-éleveurs, sa contrainte d'avancée du désert, sa population très jeune et peu éduquée, son enclavement interne et externe, un environnement écologique des plus austères et une infrastructure limitée, la commune se trouve dans l'incapacité d'améliorer sa situation de sécurité alimentaire. La question est d'autant plus cruciale que l'augmentation rapide de la population contribuera à rehausser fortement les enjeux de l'alimentation et de la nutrition. Elle a un effet aussi sur l'éducation de base, la formation, la santé, l'infrastructure de base, etc. Comment la démographie affecte-elle la nutrition et la sécurité alimentaire des ménages de la commune? Quelle possibilité de résilience s'offre-t-elle face à ce phénomène ?

\section{Demarche Methodologique}

\subsection{Cadre}

Située entre $12^{\circ} 4^{\prime} 0^{\prime \prime}$ de latitude Nord et $3^{\circ} 10^{\prime} 60^{\prime \prime}$ de longitude Est. La Commune de Karimama se trouve à la pointe septentrionale du Bénin. Son altitude moyenne est de $200 \mathrm{~m}$ au-dessus du niveau de la mer dont elle est à plus de $773 \mathrm{~km}$ à vol d'oiseau. Karimama vient d'un mot Gourmantché « Kaliman-man » qui signifie étymologiquement « là où l'on reste en paix ». Le climat de la Commune est de type sahélo-soudanien marqué par une saison sèche de Novembre à Avril. La Commune se trouve dans la vallée du Niger qui va de Kargui jusqu'au Mékrou. Le relief est dans l'ensemble peu accidenté avec une extension terminale de la chaîne de l'Atacora au Sud - ouest de la zone. Elle dispose d'une importante superficie de bas-fonds, d'un important réseau hydraulique (fleuve Niger, Mékrou, Alibori), du Parc W et d'autres aires protégées (rôneraie de Goroubi) et d'un gisement de fer à LoumbouLoumbou. La commune de Karimama, divisée en cinq arrondissements et 24 villages, occupe une superficie de $6102 \mathrm{~km} 2$ dont 40.000 ha de terres cultivables. Elle est limitée au Nord par le fleuve Niger (frontière naturelle), au Sud-ouest par la Commune de Banikoara, au Sud-est par la Commune de Kandi, à l'Est par la Commune de Malanville et à l'Ouest par le Burkina-Faso. La figure 1 présente la situation géographique de la Commune. 


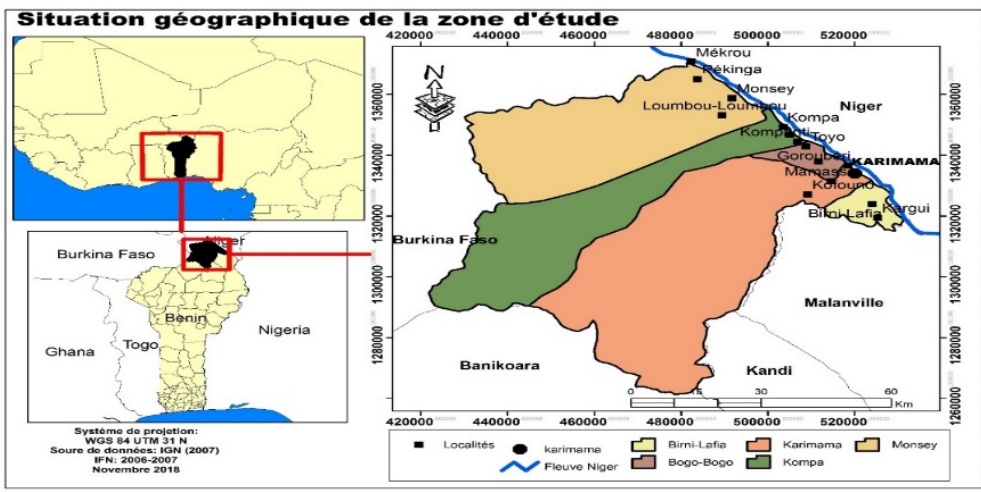

Figure1 : situation géographique de Karimama Source : réalisée sur Fond de carte d'IGN, 2007

\subsection{Types de données}

Cette étude a fait recours aux données primaires et secondaires. La collecte de données primaires a été faite auprès des ménages et au niveau de la communauté et des ménages échantillonnés. Un questionnaire ménage a servi pour l'entretien avec les chefs de ménages ou leurs représentants. Les données collectées ont porté principalement sur la croissance démographique, la sécurité alimentaire dans la commune, les sources de revenus, la consommation alimentaire, la malnutrition, les dépenses, les mécanismes d'adaptation, la diversité alimentaire, la santé et l'éducation dans la Commune. Les informations collectées ont été complétées et/ou soutenues par les données secondaires disponibles. La collecte des données secondaires a été faite à travers une revue complète des données et rapports existants relatifs aux études de la population et de la Sécurité alimentaire dans la Commune.

\subsection{Echantillonnage}

La méthode d'échantillonnage par grappes stratifiés à deux degrés a été utilisée. Chaque village constitue une strate à part entière, y compris Karimama centre. La taille de l'échantillon déterminée dans le cadre général d'une recherche en thèse permet d'assurer la représentativité des villages de la Commune. La taille minimum de l'échantillon est de 370 ménages repartis dans 09 grappes correspondant aux villages enquêtés au niveau des 5 arrondissements de la Commune. Des grappes de remplacement par strate ont été dressées afin d'identifier les options de remplacement au cas où plus de $10 \%$ des grappes initiales présentent des problèmes d'accessibilité. La taille de l'échantillon a été obtenue en utilisant l'approximation normale de la distribution binomiale proposée par Dagnelie (1998) :

$\boldsymbol{n}=\frac{\mathbf{z}^{2} * \boldsymbol{p}(\mathbf{1}-\boldsymbol{p})}{\boldsymbol{e}^{\mathbf{2}}}$ Où nous avons :

$\mathrm{n}=$ taille minimale requise de l'échantillon; 
$\mathrm{Z}=$ niveau de confiance $;$ Le seuil de confiance considéré est de $95 \%$. La valeur de 1,96 est admise pour faire les calculs $\mathrm{e}=$ marge d'erreur ; la marge d'erreur admise est de $5 \%$ $\mathrm{p}=$ taux de la malnutrition chronique des enfants de 0 à 5 ans dans la commune $(40,3 \%)$.

\subsection{Méthodes d'analyse}

L'information qualitative et subjective obtenues à travers l'observation, les entretiens individuels et les groupes de discutions a fait l'objet d'analyse de contenu. A travers les opinions, les croyances, les prises de position et les points de vue des populations, il a été apprécié les représentations sociales sur la démographie débordante de la commune ; ses relations avec la sécurité alimentaire et la malnutrition des enfants.

Les données d'enquête individuelle saisies et apurées ainsi que des données quantitatives issues de la documentation ont été analysées à l'aide du logiciel STAT 13 MP pour la détermination des statistiques descriptives en termes de pourcentage et de moyenne. Le logiciel ARCGIS 10.1 a servi à la réalisation des cartes qui ont été réalisées à l'aide du fond de carte d'IGN 2007.

Pour apprécier la couverture des besoins alimentaires dans la commune, il a été calculé le proxy calorique et comparé à la demande alimentaire. Le proxy calorique de 1996 à 2016 est calculé sur la base des informations relatives aux productions vivrières (céréales, légumineuses, racines et tubercules). Les " proxys " caloriques au niveau de la Commune ont calculé les calories disponibles per capita à partir de la production vivrière de la Commune. Ce proxy prend en compte les trois principaux groupes alimentaires, ceux qui constituent plus de $90 \%$ des apports énergétiques (c'est le tryptique « céréales / légumineuses / tubercules »). Le calcul du proxy s'est basé sur les productions nettes ${ }^{1}$ des différents produits des trois groupes, exprimées en calories, et sur la population de la Commune en année n. La norme de 2400 $\mathrm{Kcal} /$ personne/jr retenue par la FAO (2010) comme la ration alimentaire quotidienne correcte a servi de base de comparaison.

\section{Resultats}

\subsection{Dynamiques Sociodémographiques De La Commune}

\subsubsection{Structure De La Population}

Le dernier recensement de la population et de l'habitation (RGPH-4) a estimé en 2013 la population de la commune de Karimama à 66353 habitants, soit $0,66 \%$ de la population du Bénin. Les femmes représentent 50,04 \% de cette population et les hommes 49,96\% (tableau1). Le sex-ratio est de 99,8 hommes pour 100 femmes.

\footnotetext{
${ }^{1}$ La production nette prend en compte les taux de pertes et semences et les taux de transformation les plus pertinents
} 
Tableau 1: Structure de la population par sexe et par milieu de résidence en 2013

Catégorie de population

Année 2013

\section{Effectif}

Pourcentage

Population totale 66353 100

Population par sexe

$\begin{array}{ccc}-\quad \text { Homme } & 33149 & 49,96 \\ -\quad \text { Femme } & 33204 & 50,04 \\ \text { Population Urbaine } & 19707 & 29,7 \\ \text { Population Rurale } & 46646 & 70,3\end{array}$

Source : Données INSAE, RGPH-4, 2013

Le tableau 2 présente le taux d'accroissement annuel de la population de Karimama.

Tableau 2 : Taux de croissance annuel de la population de Karimama

\begin{tabular}{cccc}
\hline Niveaux & $\begin{array}{c}\text { Taux d'accroissement } \\
\text { entre 1979-1992 (en \%) }\end{array}$ & $\begin{array}{c}\text { Taux d'accroissement } \\
\text { entre 1992-2002 (en \%) }\end{array}$ & $\begin{array}{c}\text { Taux d'accroissement } \\
\text { entre 2002-2013 (en \%) }\end{array}$ \\
\hline Karimama & 2,77 & 3,13 & 4,72 \\
\hline
\end{tabular}

De $2,77 \%$ entre 1979 et 1992 , le taux d'accroissement de la population de Karimama est passé rapidement à 3,13\% entre 1992 et 2002 puis à 4,72\% entre 2002-2013.

\subsubsection{Répartition de la population par tranche d'âges}

La population de Karimama, réparties au sein des cinq (05) arrondissements de la commune est très jeune et comprend une majorité d'enfants et d'adolescents de 0 à 14 ans estimée à 33818 individus, soit 51\% de la population communale (figure 2).

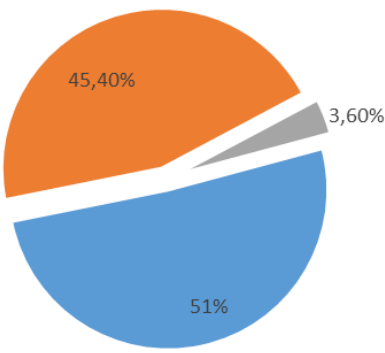

$$
\text { - } 0 \text { à } 14 \text { ans } \quad \text { - } 15 \text { à } 59 \text { ans } \quad 60 \text { ans et plus }
$$

Figure 2 : Tranche d'âges de la Population en 2013

Source : INSAE, RGPH-4, 2013 
La tranche d'âge de 0 à 14 ans, avec une proportion de $51 \%$, représente plus de la moitié de la population. Elle est suivie de la tranche d'âge de 15 à 59 ans avec une proportion de $45,4 \%$ et enfin celle de 60 ans et plus avec une proportion de $3,6 \%$.

\subsubsection{Evolution de la population dans la commune de Karimama}

Entre 1979 et 2013, la population de la Commune a connu une forte croissance passant de18123 habitants à 66353 d'habitants (figure 3).

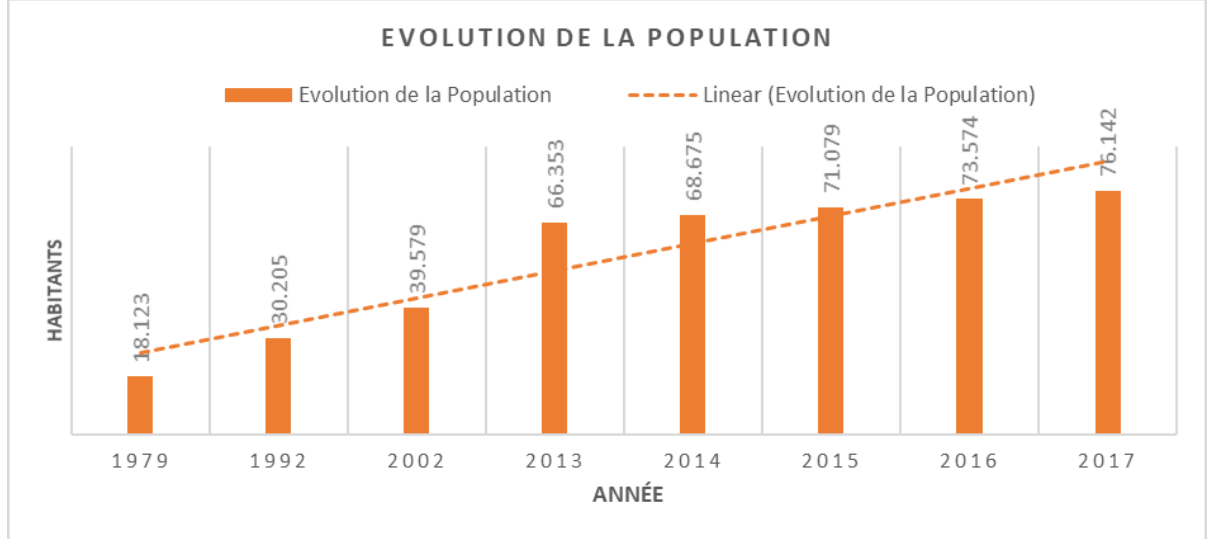

Figure 3: Evolution de la population de 1979 à 2013

Source : Données INSAE, RGPH-1, RGPH-2, RGPH-3, RGPH-4, 2013

Cette population en passant de 18123 habitants en 1979 à 76142 habitants en 2017 a été multipliée par 4,2. Ceci correspond à une croissance particulièrement importante car sur la même période, la population au niveau national n'a été multiplié que par seulement par 3. L'évolution du poids démographique de Karimama montre que sa population qui représentait 0,54 $\%$ de la population du Bénin en 1979 est passée à $0,66 \%$ en 2013 et puis à $0,69 \%$ en 2017

\subsection{Dynamique spatio-temporelle des populations}

La dynamique spatio-temporelle des populations est appréciée ici à travers l'évolution des densités de la population, des superficies des cultures et la pression exercée sur les terres qui est à l'origine de la parcellisation.

\subsubsection{Densité de la population}

En rapportant l'effectif de population recensée, 66353 habitants à la superficie exploitable disponible de la commune, $470 \mathrm{~km}^{2}$, il se dégage une densité de 141,18 habitants $/ \mathrm{km}^{2}$ en 2013 (tableau 3).

\section{-}

$1979 \quad$ Année

2002

2013 


\begin{tabular}{|c|c|c|c|c|c|c|c|c|c|}
\hline $\begin{array}{l}\text { Sup } \\
\text { totale } \\
(\mathrm{Km} 2)\end{array}$ & $\begin{array}{l}\text { Sup exploitable } \\
\text { disponible } \\
(\mathrm{Km} 2)\end{array}$ & $\begin{array}{l}\text { Pop } \\
\text { Hab. }\end{array}$ & $\begin{array}{l}\text { Densité } \\
\text { Hab. /km2 }\end{array}$ & $\begin{array}{l}\text { Pop } \\
\text { Hab. }\end{array}$ & $\begin{array}{l}\text { Densité } \\
\mathrm{Hab} \text {. } \\
\text { /km2 }\end{array}$ & $\begin{array}{l}\text { Pop } \\
\text { Hab. }\end{array}$ & $\begin{array}{l}\text { Densité } \\
\text { Hab. } \\
\text { /km2 }\end{array}$ & Pop Hab. & $\begin{array}{l}\text { Densité } \\
\mathrm{Hab} . / \mathrm{km} 2\end{array}$ \\
\hline 6041 & 470 & 18123 & 38,56 & 30205 & 64,27 & 39579 & 84,21 & 66353 & 141,18 \\
\hline
\end{tabular}

\subsubsection{Parc $W$ et occupation du terroir}

La Réserve Transfrontalière de Biosphère du W (RTBW) occupe les cinq sixième $(5 / 6)$ de la superficie totale de la commune (Figure 4).

Figure 4 : Le Parc National du W et la mosaïque d'habitation, de culture et de Jachère

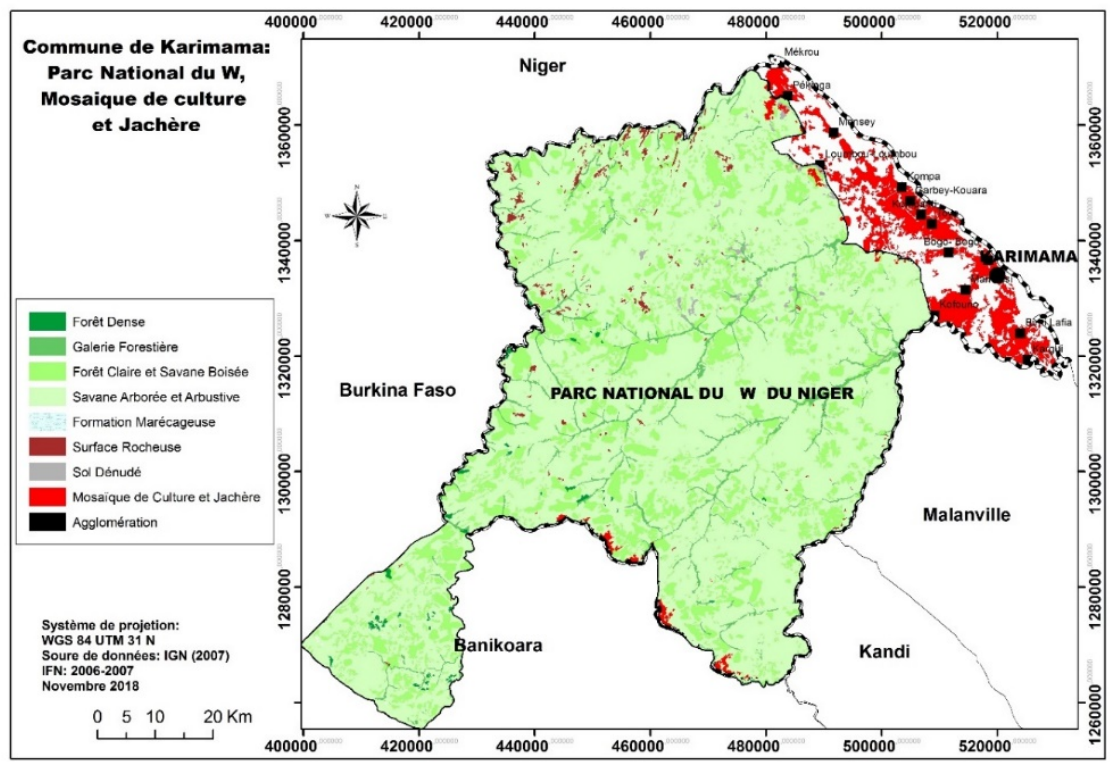

Source : Réalisée à partir du Fond de carte de l'IGN

Le Parc W, une zone protégée, réduit la zone " habitable » de la Commune à une bande d'au plus $15 \mathrm{~km}$ de large le long du fleuve Niger, 470 $\mathrm{km}^{2}$ des $6102 \mathrm{~km}^{2}$ de la superficie totale. L'espace non occupé par le parc W est couvert par des habitations, des champs de cultures, des jachères, des savanes arbustives et des tâches de forêts galerie (PDC 3, 2017). Le domaine agricole (champs de culture et jachères) s'agrandit très peu malgré la pression démographique et la culture extensive pratiquée dans la commune.

\subsubsection{Occupation humaine et mosaïque de cultures}

La dynamique de l'occupation du sol permet d'apprécier la répartition des terres entre l'habitat humain, l'agriculture et les zones protégées. En raison de la présence du parc $\mathrm{W}$, les zones protégées occupent plus de $5 / 6$ de la superficie de la commune. L'île aux oiseaux occupe quant à elle 13000 ha de terre (PDC 3, 2017). Traversée également par le fleuve Niger et ses affluents l'Alibori et Mekrou, les terres inondables et bas-fonds aménageables de la 
commune occupent par ailleurs une superficie de 14230 ha de terre. Finalement, les zones d'occupations humaines, de reboisement de rente ou forestier, des sols dénudés ou végétation clairsemée et de la savane arborée et des aires agricoles ne recouvrent qu'une infime partie de l'espace communal.

\subsubsection{Evolution de la production agricole de la commune de Karimama}

Les superficies des cultures dans la commune ont très peu évolué ces 20 dernières années comme le montre la figure 5 .

Figure 5 : Evolution des superficies emblavées des différentes cultures ces 20 dernières années (1996-2016)

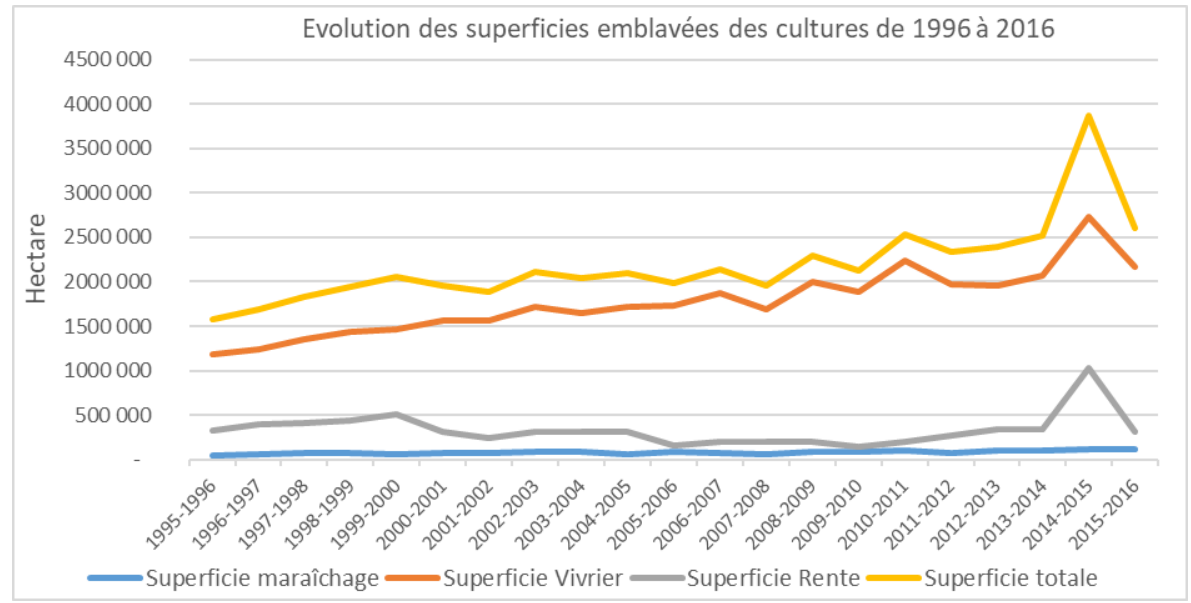

Source : Données DSA/DDP-MAEP

\subsection{Dynamique démographique et performance agricole}

Les rythmes de croissance démographiques sont de loin plus élevés que ceux de de la production. La croissance démographique fait ainsi peser de lourdes incertitudes sur la capacité de la commune à nourrir sa population. Elle crée de nouveaux enjeux sur lesquels, il faudrait se pencher au risque de laisser se développer ou s'aggraver des fragilités.

\subsubsection{Production agricoles et rendement des cultures}

L'économie de la commune de Karimama est essentiellement basée sur le secteur agricole. Les principales activités menées sont: l'agriculture, l'élevage et la pêche. L'agriculture est la principale source de revenu des populations. Les principales cultures pratiquées sont les cultures vivrières (sorgho, petit mil, riz, maïs, niébé), les cultures maraîchères (oignon, tomate, pomme de terre, piment, gombo) et les cultures de rente (coton, arachide). La figure 6 présente l'évolution des productions des cultures et leurs rendements dans la période de 1996 à 2016. 


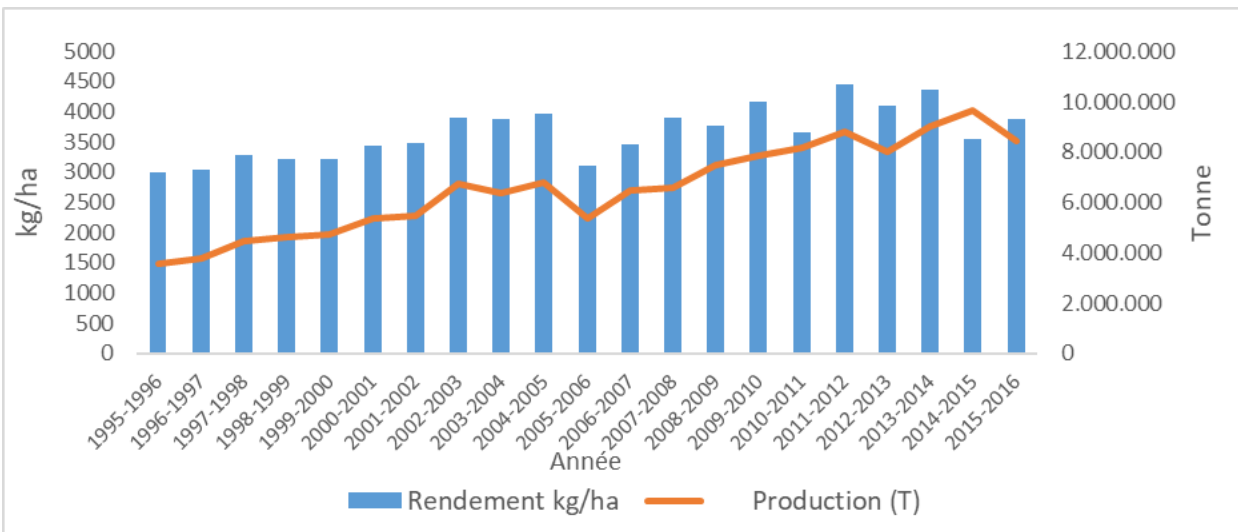

Figure 6: Evolution de la production et du rendement des cultures entre (1996-2016) Source : données DSA/DPP-MAEP

\subsubsection{Croissance démographique et croissance agricole}

Pour maintenir ou améliorer le niveau des rations alimentaires, les taux de croissance agricole surtout vivrier doivent être plus élevés que ceux démographiques. Ils permettent également de redresser une situation alimentaire grave. La figure 7 montre, les taux de croissance agricoles, comparés à ceux démographiques.

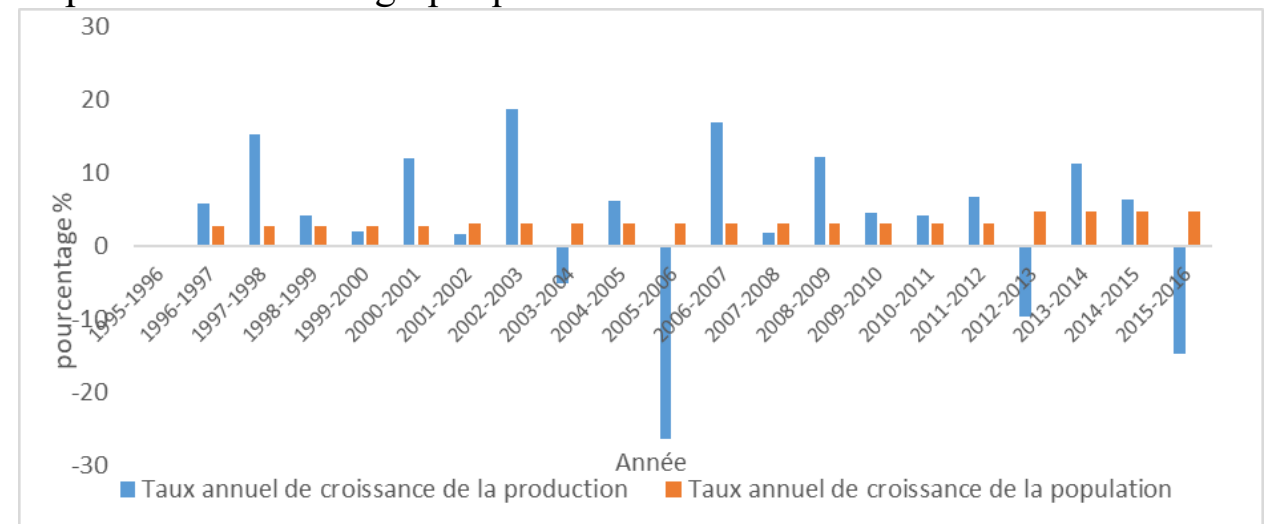

Figure 7: Evolution des taux annuels d'accroissement de la population et de la production agricole

Source : données DSA/DPP-MAEP

\subsubsection{Croissance démographique et disponibilité alimentaire}

Le rythme de croissance démographique qui se fait sans répit augmente la demande d'aliments et nécessite une production supplémentaire des vivres. Lorsque ce surplus n'est pas produit, l'offre devient inférieure à la demande et se traduit par une couverture insuffisante des besoins alimentaires. Pour apprécier la couverture des besoins alimentaires dans la commune, il a été calculé le proxy calorique et comparé à la demande alimentaire (Figure 8). Le proxy calorique est calculé dans la période de 1996 à 2016 sur la base des 
informations relatives aux productions vivrières (céréales, légumineuses, racines et tubercules).

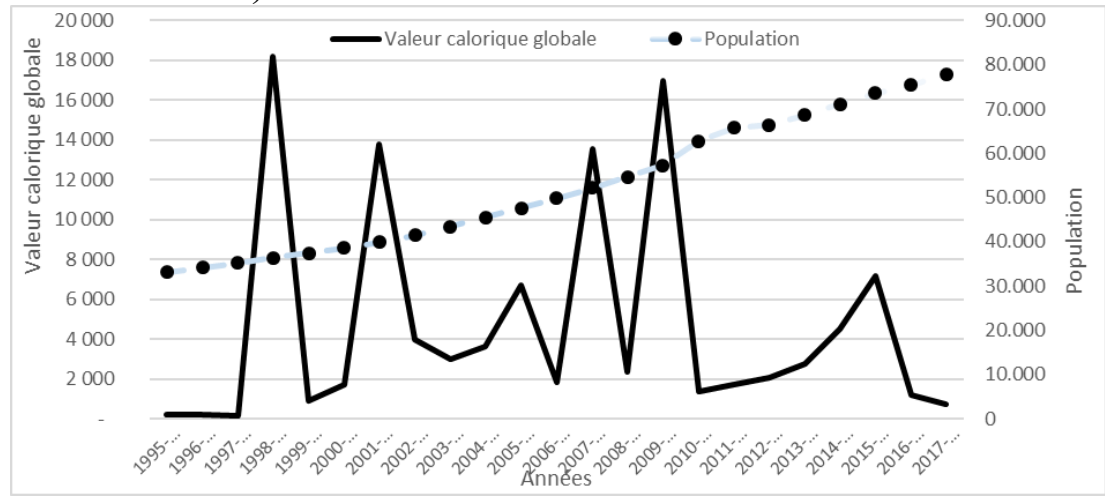

Figure 8: Evolution de la production des denrées alimentaires comparée à la population Source: données MAEP/DSA, 2019

\subsection{Situation nutritionnelle et accès aux services sociaux}

En dehors de la demande alimentaire, l'augmentation de la population crée des besoins en logements, eau, hygiène, éducation, soins nutritionnel et de santé.

\subsubsection{Situation Nutritionnelle des populations dans la commune}

Tableau 4: Niveaux et tendances de la Malnutrition des enfants de 6-59 mois

\begin{tabular}{cccccc}
\hline $\begin{array}{c}\text { Différentes formes de } \\
\text { malnutrition }\end{array}$ & \multicolumn{2}{c}{ PNC } & CAP & PMASN & $\begin{array}{c}\text { Norme } \\
\text { OMS }\end{array}$ \\
\cline { 2 - 6 } & 2011 & 2015 & 2016 & 2018 & \\
\hline $\begin{array}{c}\text { Malnutrition aigüe ou } \\
\text { Emaciation }\end{array}$ & 22,92 & 16,7 & 15,1 & 13,0 & $<5 \%$ \\
\hline $\begin{array}{c}\text { Insuffisance pondérale } \\
\text { Malnutrition chronique ou } \\
\text { Retard de croissance }\end{array}$ & 47,92 & 38,0 & 32,9 & 30,8 & $<10 \%$ \\
\hline
\end{tabular}

Source: Données PNC, 2015, Etude CAP UNICEF et PMASN

\subsubsection{Accès aux services de santé}

Le secteur de la santé dans la commune de Karimama est confronté au manque de personnel soignant, à la vétusté du plateau technique, aux difficultés d'accès aux soins et aux centres de santé, au manque de produits pharmaceutiques dans les centres de santé de la zone rurale. La commune toute entière ne dispose que de six (06) formations sanitaires composées d'un (01) centre communal de santé (CCS) et de cinq (05) dispensaires et unités villageoises de santé. Le personnel exerçant est constitué d'une infirmière, d'un infirmier, d'une sage-femme et d'un médecin. Il n'y a ni laboratoire, ni technicien pour les analyses de sang. En 2018, la commune comptait un seul médecin pour plus de 76000 habitants alors qu'au niveau national le ratio habitants/médecin est de 7 979. La couverture de l'offre des services de santé 
au niveau local, généralement mesuré par la proportion de la population ayant accès à un service de santé dans un rayon de $5 \mathrm{~km}$, demeure très faible (moins de $30 \%$ ). Le taux de fréquentation des services de santé est aussi faible et tourne autour de $35 \%$. En ce qui concerne, le ratio habitants par infirmier, il est à plus 38000 largement supérieur à la norme de 5000 recommandée par l'OMS. Le ratio Femme en Age de Procréer/sage-femme est aussi très élevé et est à plus de 25000 par rapport 1563 au niveau national. Avec une prévalence contraceptive de $18 \%$ en 2014 (MICS) et à peine $5 \%$ à Karimama, le Bénin est encore loin d'avoir réalisé sa «révolution contraceptive».

\subsubsection{Mortalité des enfants}

Le contexte de la santé dans la commune de Karimama reste marqué par la faible réduction de la mortalité maternelle et infanto-juvénile (Tableau 5).

Tableau 5 : Quotients de mortalité infantile, juvénile et infanto-juvénile selon le milieu de résidence et le département

\begin{tabular}{cccc}
\hline \multicolumn{2}{c}{ Année Enquête EDS-B } & \multicolumn{2}{c}{ Niveaux } \\
\hline & EDS-III Bénin 2006 & Département Alibori & National \\
\hline$-\quad$ Mortalité infantile (1q0) & 86 & $\mathbf{7 6}$ \\
\cline { 2 - 3 }$-\quad$ Mortalité juvénile (4q1 & 85 & $\mathbf{6 4}$ \\
\cline { 2 - 3 }$-\quad$ Mortalité infanto-juvénile (5q0) & 164 & $\mathbf{1 3 6}$ \\
\hline EDS-IV Bénin 2011-2012 & & $\mathbf{4 5}$ \\
\hline$-\quad$ Mortalité infantile (1q0) & 58 & $\mathbf{3 1}$ \\
\cline { 2 - 3 }$-\quad$ Mortalité juvénile (4q1 & 56 & $\mathbf{7 5}$ \\
\hline & EDS-V Bénin 2017-2018 & 111 & 55 \\
\hline & Mortalité infanto-juvénile (5q0) infantile (1q0) & & 44 \\
\cline { 2 - 3 }$-\quad$ Mortalité juvénile (4q1 & $\mathrm{Nd}$ & 96
\end{tabular}

Source : INSAE/EDSB, 2017-2018

Au niveau national, en 2018, le risque de mortalité infantile, est évalué à 55 décès pour 1000 naissances vivantes et le risque de mortalité juvénile à 44 décès pour 1000 . Les 2 composantes de la mortalité infantile, que sont la mortalité néonatale et la mortalité post néonatale, sont estimées respectivement à 30 décès pour 1000 et à 25 décès pour 1000 . Le poids de la mortalité néonatale dans la mortalité infantile est plus important que celui de la mortalité post néonatale (55\% par rapport à $45 \%$ ). Globalement, le risque de mortalité infanto-juvénile, c'est à dire le risque de décès avant l'âge de 5 ans, est de $96 \%$. Ce risque doit être encore plus élevé au niveau départemental et communal puisqu'en 2012, il était respectivement de 58 décès pour 1000 et 56 décès pour 1000 au niveau du département.

La fécondité reste élevée au Bénin. L'Indice Synthétique de Fécondité (ISF) est estimé à 5,7 enfants par femme, au niveau national. Avec un écart de 
près d'un enfant par femme, l'ISF est plus élevé en milieu rural qu'en milieu urbain. Il est de 6,1 enfants par femme dans le département de l'Alibori qui abrite la commune de Karimama

\section{Analyse}

Avec un taux annuel d'accroissement de la population de 3,5\% et une population qui s'accroît de quelque 300000 personnes par an (RGPH-4, 2013), le Bénin est l'un des pays au sud du Sahara qui est loin d'achever sa transition démographique. Karimama, une des Communes rurales du Bénin présente un fort taux d'accroissement annuel de la population de $4,72 \%$ en 2013. La commune a vu multiplier sa population par 3,66 entre 1979 et 2013 passant de 18123 habitants à 66353 habitants. Cette démographie débordante entraîne un déséquilibre énorme entre la production des vivres et les besoins alimentaires. Elle représente aussi de grands risques de vulnérabilité à la malnutrition des enfants et de prise en charge de multiples besoins sociaux pour les populations. Avec un sex-ratio de 99,8 hommes pour 100 femmes, la population de Karimama est composée de plus de $51 \%$ de jeunes de la tranche d'âge de 0 à 14 ans. Ce qui est redouté dans une population à majorité jeune, ce sont les conséquences sociales de la charge accrue des jeunes âgés de moins de 15 ans et du volume annuel important des enfants de moins de cinq ans à nourrir et soigner. La population de Karimama est concentrée sur une bande très réduite de la superficie de la Commune. Cette concentration des habitants sur cette bande fait de Karimama l'une des plus fortes densités de la population du Pays. L'analyse spatio-temporelle montre que la densité de la population est passée de 38,56 habitants/Km2 à 141,18 habitants/Km2 entre 1979 et 2013; sur une même superficie de terre, la densité de la population a été multipliée par quatre en 35 ans environ. A Karimama, la forte densité de la population s'explique aussi par l'occupation d'une bonne partie du territoire par le Parc W (la Réserve Transfrontalière de Biosphère du W). Ainsi, le Parc W et l'accroissement constant de la population renforcent fatalement la pression sur la terre. Cette surcharge croissante du sol devient encore plus préoccupante sachant qu'elle limite la production alimentaire. C'est à juste titre que l'UNFPA déclarait que « tous les pays, en particulier les plus pauvres, doivent s'appuyer sur des données et des prévisions démographiques pour éclairer leurs stratégies de développement »(UNFPA, 2011b). Les agriculteurs sont ainsi confrontés au problème de terres cultivables, à l'appauvrissement croissant des terres parce que surexploitées et à la concurrence pour l'exploitation des ressources naturelles. La tendance au morcellement des terres, à l'augmentation du nombre de petits et très petits exploitants se manifeste aussi nettement dans la commune. Ceci constitue un problème pour une population agricole et une Commune considérée comme une des portes d'entrée du désert au Bénin et de la transhumance transfrontalière. A 
Karimama, on observe une faible variation à la hausse des superficies emblavées au niveau de toutes les cultures (vivrières, maraichères et de rente). Mais cette hausse bien que faible se fait souvent aux dépens des prairies et pâturages permanents (PDC 3, 2017). L'insuffisance d'espace cultivable dans la commune contraint les agriculteurs à exploiter d'une part, les terres inondables du fleuve Niger et de ses affluents et d'autre part, les mêmes terres pendant des années quitte à appauvrir totalement les sols. L'utilisation répétée sans jachère réduit le potentiel productif agricole de la commune. En effet, dans la Commune, près de $80 \%$ des terres (mosaïque, champ et jachère) sont caractérisées par des sols avec un potentiel agricole faible ou moyen. L'érosion des sols et leur dégradation due à l'impact des activités humaines et la faible teneur en carbone organique des couches arables limitent à $13 \%$ en moyenne les terres à fort potentiel. Le rendement des principales cultures traduit bien la faible qualité agro-environnementale de la Commune. Entre 1996 et 2016, les productions agricoles ont peu augmenté et les rendements peu varié. Cette faible variation à la hausse des productions est due plus à l'augmentation de la surface récoltée. Pour augmenter la production des cultures, les agriculteurs étendent les superficies emblavées dans un esprit de culture extensive. Dans un contexte de forte croissance démographique, cette agriculture rustique de type extensif aura pour conséquence d'augmenter à la fois le nombre de paysans sans terre et la surexploitation des terres. Le nombre d'exploitants agricoles augmente alors que les surfaces foncières ne s'accroissent pas. La conséquence immédiate est l'insuffisance des productions vivrières et l'incapacité des producteurs à se nourrir par leur propre production. Pour que les objectifs mondiaux en matière de sécurité alimentaire puissent être atteints, les populations rurales devraient avoir à leur disposition des terres suffisantes pour produire de plus en plus de nourriture de qualité, tout en améliorant leur situation économique » (FAO, 2005). Dans la Commune, le taux annuel d'accroissement de la population est en constante augmentation alors que celui de la production agricole a une évolution en dent de scie. Parfois ces taux prennent des valeurs négatives, c'est le cas en 20032004, 2005-2006, 2012-2013 et 2015-2016, traduisant une production alimentaire instable pour la consommation d'une population sans cesse croissante. La production alimentaire disponible pour la consommation est presque toujours largement inférieure aux besoins de la population. La norme de $2400 \mathrm{Kcal} /$ personne/jr $(\mathrm{FAO}, 2010)$ de la ration alimentaire quotidienne correcte reste encore une équation dans la commune. Cette ration ne donne aucune garantie d'une année à l'autre. En effet, de $195 \mathrm{kcal} / \mathrm{hab}$./jour entre 1995-1996; elle passa subitement à $18184 \mathrm{kcal} / \mathrm{hab} . /$ jour entre1998-1999 avant de rechuter à $917 \mathrm{kcal} / \mathrm{hab}$./jour entre 1999-2000. L'évolution du disponible alimentaire comparée à celle de la population indique un écart marqué entre la production alimentaire et la demande réelle. Cet écart fait 
ressortir la gravité de la sous-alimentation se caractérisant par une insuffisance quantitative et qualitative des denrées alimentaires dans la commune. Sous l'influence de la pression démographique, l'écart pourrait à long terme avoir une tendance exponentielle avec comme conséquence un bilan qui sera toujours négatif. Comme les activités agricoles représentent la principale composante des moyens d'existence des populations de la commune, ces dernières verraient leurs moyens d'existence réduits et leur accès aux vivres limité. Les ménages en général et les pauvres en particulier, auront à arbitrer entre les besoins fondamentaux alimentaires et non alimentaires. Ce qui suscite de sérieuses interrogations sur la satisfaction des besoins alimentaires en fonction des normes recommandées (FAO, 2010).

Les enfants sont assez souvent les premières victimes de l'insécurité alimentaire qui les fragilise et les rende vulnérables à la malnutrition. Dans la commune, la prévalence de la malnutrition sous toutes ses formes (Emaciation, retard de croissance et insuffisance pondérale) au sein des enfants de moins de cinq (05) ans est élevée et dépasse les seuils critiques suggérés par l'OMS. L'analyse des données nutritionnelles de la commune montre que la malnutrition est encore à un niveau inacceptable. En 2018, 30,8 $\%$ des enfants de moins de 5 ans souffraient d'insuffisance pondérale, 13,0\% d'émaciation et $40,3 \%$ de retard de croissance. Par rapport aux normes de l'OMS, cette situation nutritionnelle est très critique car le risque de mourir est très élevé lorsque la malnutrition est sévère (D.L. Pelletier, 1994). Au Niger, la malnutrition aiguë des enfants est la plus forte pendant la période hivernale de semence, en saison de pluie, quand les stocks de céréales sont épuisés dans les greniers (R. Ndamobissi, 2017). L'état nutritionnel des enfants de moins de 5 ans d'une communauté reflète souvent la situation alimentaire et nutritionnelle de cette dernière (EDS, 2017-2018). Le taux de malnutrition exprime à lui seul la complexité et le caractère multifactoriel de la sécurité alimentaire et nutritionnelle (OCDE, 2012). De façon générale, l'état de santé de la population, suivant les conditions de vie actuelle, est assez précaire. Les services de santé locaux manquent souvent de personnel et de médicaments essentiels. L'attitude à l'égard des soins de santé et de l'éducation est un autre facteur important de la propension des mères à recourir aux services mis à leur disposition. Ces phénomènes sont encore aggravés par l'accroissement rapide de la population (FAO/OMS, 1992 d). La présence des services de santé et leur facilité d'accès influence le traitement de maladies et la prévalence de malnutrition qui est responsable des cas de décès qui surviennent avant l'âge de 05 ans.

Les relations entre les paramètres démographiques et la production alimentaire ont depuis toujours été d'un grand intérêt pour l'humanité. $\mathrm{Au}$ XVIIIe siècle déjà, Thomas R. Malthus s'était fait remarquer par son postulat concernant les risques démographiques de la famine occasionnée par une 
croissance rapide de la population (J-P. Marechal, 1963). Thomas R. Malthus (1798) part du postulat selon lequel: « la population croit à un rythme géométrique (plus rapide) que la production alimentaire qui croit à un rythme arithmétique ». Dans sa publication "Essai sur le principe de population", il écrivait : «La race humaine croîtra selon la progression 1, 2, 4, 8, 16, 32, 64, $128,256 .$. tandis que les moyens de subsistance croîtront selon la progression $1,2,3,4,5,6,7,8,9$. Au bout de deux siècles, population et moyens de subsistance seront dans le rapport de 256 à 9 ; au bout de trois siècles, 4096 à 13 ; après deux mille ans, la différence sera immense et incalculable » $(\mathrm{H}$. Theil, 1963, p12). Dès lors, le déterminisme démographique de Malthus a orienté les approches des problèmes de croissance démographique et alimentaire. M. TRAORE (2010, p.69) dira que « la poussée démographique qui réduit le volume des stocks disponibles et annuellement renouvelables de produits alimentaires par habitant, accentue la dépendance des pays africains par rapport à l'importation des vivres. La situation exige des mesures urgentes pour diminuer cette dépendance $»$. Mais les solutions apportées aux problèmes alimentaires à travers d'une part, la révolution agricole avec la mécanisation, la fertilisation des sols, la baisse des prix et les facilités d'accès aux produits alimentaires et d'autre part, la transition démographique ont tôt faire de relativiser la théorie de Malthus. L'approche du « déclin dans la disponibilité alimentaire » stipule que la famine est causée par une réduction drastique de la nourriture par tête résultant d'un « choc exogène ». La baisse de la ration alimentaire individuelle et donc de la consommation calorifique va entraîner la malnutrition des enfants (Rossel, 2008) et accroître la mortalité des enfants et des adultes à cause de la faim et des maladies infectieuses. Ce modèle a été bien vécu dans le cas de la grande famine des années 1846-1848 survenue en Irlande (R. S. Walter \& Schofield, 1989). Ensuite l'approche par le déclin des politiques alimentaires qui se penche sur le système politique (leadership, instabilité, mal gouvernance, etc.) et les défaillances des politiques stratégiques parmi lesquels on peut noter : i) la théorie de la sous-information et aussi manque de liberté (S. Amartya, 1999), ii) les conditions de gouvernance politique qui prédisposent le pays à une famine ou la malnutrition des enfants (IDS, 2012). Les contraintes institutionnelles, administratives, logistiques ou d'infrastructures ont toujours été considérées comme étant des obstacles majeurs par les pays qui subissent la famine. Il s'agit des facteurs structurels de sous-développement qui traduisent les faibles investissements publics dans les infrastructures (Banque Mondiale, 2000). La famine est alors perçue comme une conséquence de la défaillance politique plutôt que des problèmes économiques. Ainsi, ce sont les politiques qui sont conduites actuellement qui vont déterminer si la population mondiale va excéder 9 milliards de personnes en 2050, puis se stabiliser aux alentours de 10 milliards en 2100, ou au contraire dépasser les 10 milliards d'individus vers le milieu 
du siècle et être portée à 16 milliards à la fin du siècle. L'écart entre les prévisions moyennes et hautes des Nations unies concernant la population correspond à 0.5 enfant de plus qu'aujourd'hui par femme et par an en moyenne (UNFPA, 2011b ; Nations unies, 2011). Chaque décennie de retard sur l'objectif du seuil de remplacement à atteindre se traduit par une croissance démographique notable et soutenue dans les décennies à venir (Nations unies, 2011). Alors, la poussée démographique seule n'explique pas les problèmes alimentaires et nutritionnels que connait le monde. Une revue de la littérature qui a investi cette question de l'évolution conjointe des trois déterminants de la sécurité alimentaire que sont la population, la situation alimentaire et les ressources disponibles, a rapidement fait émerger le constat selon lequel cette conjonction n'est pas linéaire et qu'elle constitue une condition nécessaire mais non suffisante pour réduire l'insécurité alimentaire (FAO, 2017 ; FAO, FIDA, PAM, 2012). C'est pour cela que « les efforts destinés à encourager l'adoption de trajectoires de développement plus durables doivent tenir compte des populations, de leur importance, de leur situation géographique et de leur structure selon l'âge, ainsi que de leurs conditions de vie, de leurs ambitions et des perspectives qui s'offrent à elles » (IIASA, 2011). Il apparaît ainsi l'approche du dividende démographique qui est le bénéfice que peut retirer un pays du changement de sa structure par âge au cours de la période unique de son histoire que constitue la transition démographique. David E. Bloom et Jeffrey G. Williamson de l'Université de Harward ont pu mettre en exergue la contribution très positive de la transition démographique spectaculaire intervenue en Asie au vingtième siècle caractérisée par une baisse très rapide de la mortalité et de la fécondité et un afflux de la main d'œuvre qualifiée jeune ayant pu bénéficier des formations adéquates, au succès du miracle économique intervenu dans les pays asiatiques tels que la Chine, Hong Kong, Japon, Corée du Sud, Singapour, Taiwan, etc. (D.E. Bloom \& J.G. Williamson, 1998). Il existe donc des solutions à chaque défi démographique identifié. La pression démographique a engendré l'adoption des systèmes très intensifs de production agricole en augmentant les fréquences des récoltes sur une même superficie. Par contre, la faible densité démographique très répandue en Afrique entretient la culture extensive à faible rendement marquée par la pratique de la jachère du sol (Ester. Boserup, 1970). UNFPA, (2011a) affirmait qu'en 2050, la population des pays sousdéveloppés aura doublé et la main d'œuvre disponible totale continuera de progresser sous l'effet de l'arrivée d'environ 33000 jeunes sur le marché du travail chaque jour. Les solutions de l'insécurité alimentaire et de la malnutrition ne relèvent pas de la seule limitation des naissances. Certes, la baisse de la fécondité réduira temporairement les taux de dépendance et ouvrira aux ménages et aux pays la possibilité d'intensifier leurs investissements dans des ressources productives (CIPD, 1994, paragraphe 
3.14). Mais même si les taux de fécondité étaient ramenés rapidement au niveau de remplacement, les populations continueraient d'augmenter pendant des décennies en raison simplement du nombre de femmes en âge de procréer (OCDE, 2012). Elles dépendent de décisions dans l'agriculture, la recherche, le commerce, la santé et bien d'autres domaines. Une meilleure gouvernance et l'extension des ressources agricoles peuvent contrebalancer la vision alarmiste de peuplement et rendre le facteur démographique positif pour le développement contextualisé des pays (Y. Charbit \& M. Gaimard, 2015). La commune de Karimama devra trouver un moyen pour faire de son importante jeunesse un atout pour le développement futur de la commune et non une source d'insécurité alimentaire, de tension et de pauvreté. Il faut: i) garantir l'accès de tous aux soins de santé sexuelle et génésique ; ii) réaliser des investissements dans l'éducation, spécifiquement axés sur la parité entre les sexes; iii) trouver des moyens de rendre les femmes autonomes; et iv) veiller systématiquement à prendre en compte les prévisions démographiques dans les stratégies et les politiques de développement (OCDE, 2012). Investir davantage, par exemple, dans la jeunesse permettra de pouvoir compter sur une main-d'œuvre en meilleure santé, mieux formée et plus productive ; si les jeunes trouvent des emplois, il en résultera une croissance économique plus forte et plus soutenue. Un capital humain plus développé et un environnement social et écologique favorable serait un gage pour la réduction des conséquences sociales de la charge accrue de la population.

\section{Conclusion}

Les enjeux démographiques impactent chaque année un peu plus la commune de Karimama en fragilisant différents domaines de la vie des populations. Il a été constaté, à travers plusieurs méthodes d'analyse croisée, que l'accroissement continu et considérable de la population exacerbe les besoins alimentaires alors que la compétition pour l'accès aux terres agricoles n'a jamais été aussi vive. La résilience à l'insécurité alimentaire des ménages vulnérables dans la commune requiert des efforts importants dans la réduction de la malnutrition des enfants, l'accès des femmes à la contraception pour la maîtrise de leur fécondité, l'accès services adéquats de santé, à l'eau et aux infrastructures de production. La reconnaissance de la dimension démographique du développement est nécessaire pour la lutte contre l'insécurité alimentaire et la pauvreté dans la Commune. Car si la Commune ne planifie pas sa transition démographiques et ne profite pas des avantages qui en résultent, elle se retrouvera dans un comportement de sapeurspompiers, en réagissant de façon coûteuse et peut-être inefficace aux problèmes démographiques L'évolution démographique peut ouvrir des perspectives nouvelles pour le développement durable. Les interrelations qui existent entre émergence, transition démographique, dividende 
démographique et révolution contraceptive ne sont-elles pas des opportunités à saisir ?

\section{References:}

1. BIT. (2011) : «Croissance, emploi et travail décent dans les pays les moins avancés », rapport du Bureau international du travail pour la Quatrième Conférence sur les pays les moins avancés, Istanbul, 9-13 mai 2011, BIT, Genève.

2. Bloom, D. E., \& Williamson, J.G. (1998): Demographic Transitions and Economic Miracles in Emerging Asia. Revue Economique de la Banque Mondiale, 12, pp. 419-455.

3. Charbit Y. \& Gaimard M. (2015) : La Bombe Démographique en Question. Paris: Presses Universitaires Françaises.

4. Conférence internationale sur la population et le développement (CIPD). (1994) : « Programme d'action », rapport de la Conférence internationale sur la population et le développement, 5-13 septembre 1994, UNFPA, Le Caire.

5. Conférence des Nations unies sur les échanges et le développement (CNUCED) (2011) : « Rapport 2011 sur les pays les moins avancés : Contribution potentielle de la coopération Sud-Sud à un développement équitable et durable », CNUCED, Genève et New York.

6. CILSS. (2004) : Normes de consommation des principaux produits alimentaires dans les pays du CILSS [En ligne]. Niamey, AGRHYMET, 67

pages.

Disponible

sur : http://www.hubrural.org/IMG/pdf/cilss_rapport_normes_conso_ alimentaires.pdf (consulté le 11/07/2017).

7. CSA. (2016) : Urbanisation, transformation des zones rurales et incidences sur la sécurité alimentaire et la nutrition. Principaux domaines nécessitant une attention au niveau des politiques et rôle possible du CSA (y compris projet de décision) [En ligne]. Rome, FAO, Quarante-troisième session « Sécurité alimentaire et nutrition faire la différence $», \quad 21$ pages. Disponible sur: http:/www.fao.org/3/a-mr205f.pdf(consulté le 28/02/2017).

8. DURY Sandrine, BOCOUM Ibrahima. (2012) : Le «paradoxe » de Sikasso (Mali) : pourquoi « produire plus » ne suffit-il pas pour bien nourrir les enfants des familles d'agriculteurs [En ligne]. Cahiers Agricultures, vol. 21, n॰5 (pp. 324-336). Disponible sur : http://revues.cirad.fr/index.php/cahiers- 
agricultures/article/download/30989/30749 (consulté le 20/02/2017). DOI : 10.1684/agr.2012.0584.

9. FAO. (2010) : Produire davantage pour nourrir 9 milliards d'individus, FAO, Rome.

10. FAO. (2005) : Les femmes et le droit foncier : Sur un pied d'égalité

11. FAO. (1996) : Besoins alimentaires et croissance démographique [En ligne]. In FAO. Sommet mondial de l'alimentation, Rome, FAO-DES, Documents d'information technique, volume 1, chapitre 4 Disponible sur : http://www.fao.org/docrep/003/W2612F/w2612f04a.htm (consu lté le 11/07/2017).

12. FAO. (1996a): Atlas technique. Importance économique de l'agriculture [En ligne]. In FAO. Sommet mondial de l'alimentation, Rome, FAO-DES, Documents d'information technique, volume 1, chapitre 15 Disponible sur : http://www.fao.org/docrep/003/W2612F/w2612fMap06f.pdf (consulté le 13/07/2017).

13. FAO. (1999): Principales tendances de la production agricole mondiale, de la demande, du commerce et de la sécurité alimentaire [En ligne]. In FAO. L'agriculture, le commerce et la sécurité alimentaire: questions et alternatives concernant les prochaines négociations de l'organisation mondiale du commerce dans la perspective des pays en développement, Genève, FAO-ESC, document $\quad \mathrm{n}^{\circ} 1$. Disponible sur: $\mathrm{http}: / \mathrm{www}$.fao.org/3/ax2996f/index.html (consulté le 28/02/2017).

14. FAO. (2001) : Les treize pays qui ont le mieux réussi à réduire la sousalimentation, 1980-1997 [En ligne]. In FAO. Cent vingtième session du Conseil de la FAO, Rome, FAO-SSC, CL 120/INF/18. Disponible sur : http://www.fao.org/3/a-y0866f/index.html (consulté le 28/02/2017).

15. FAO. (2015) : L'après 2015 et les ODD: Nourrir les êtres humains, prendre soin de la planète [En ligne], Rome, FAO, n ${ }^{\circ} 14,28 p$; Disponible sur: http://www.fao.org/3/a-az775f.pdf(consulté le 28/02/2017).

16. FAO. (2015a) : Vue d'ensemble régionale de l'insécurité alimentaire en Afrique: Des perspectives plus favorables que jamais [En ligne], Accra, FAO-RAF, 39 pages ; Disponible sur : http://www.fao.org/3/ai4635f.pdf (consulté le 28/07/2017).

17. FAO. (2016) : Les indicateurs de la sécurité alimentaire [En ligne], In FAO-ESS. Statistiques de sécurité alimentaire, Rome, FAO-ESS ; Disponible sur: http://www.fao.org/economic/ess/ess-fs/indicateursde-la-securite-alimentaire/fr (consulté le 28/02/2017). 
18. FAO. (2017): L'avenir de l'alimentation et de l'agriculture. Tendances et défis. Résumé [En ligne], Rome, FAO, 47p. Disponible sur : http://www.fao.org/3/a-i6881 f.pdf (consulté le 28/02/2017).

19. FAO, FIDA, PAM. (2012) : L'état de l'insécurité alimentaire dans le monde 2012. La croissance économique est nécessaire mais elle n'est pas suffisante pour accélérer la réduction de la faim et de la malnutrition [En ligne], Rome, FAO, 73p; Disponible sur : http://www.fao.org/docrep/017/i3027f/i3027f.pdf(consulté le 15/07/2017).

20. FAO, FIDA, PAM. (2015) : L'état de l'insécurité alimentaire dans le monde. Objectifs internationaux 2015 de réduction de la faim : des progrès inégaux [En ligne], Rome, FAO, 66 pages. Disponible sur : http://www.fao.org/3/a-i4646f.pdf (consulté le 19/02/2017).

21. Fonds des Nations Unies pour la population (UNFPA). (2011a) : « Population Dynamics in the Least Developed Countries: Challenges and Opportunities for Development and Poverty Reduction ", document présenté à la Quatrième Conférence sur les pays les moins avancés, Istanbul, 9-13 mai 2011, UNFPA, New York.

22. Forum économique mondial (FEM) et Fonds des Nations unies pour la population (UNFPA). (2012): "The Missing Link in Sustainable Development: A Call to Integrate Population in the Water, Food, Energy Nexus », Statement of a Global Expert Panel, mars 2012, UNFPA, New York.

23. Herrmann, Michael. (2012). «Faire de la dynamique démographique un paramètre du développement durable », dans OCDE, Development Co-operation Report 2012 : Lessons in Linking Sustainability and Development, Éditions OCDE, Paris.

24. IDS. (2012): 'Fighting Maternal and Child Malnutrition: Analysing the political and institutional determinants of delivering a national multi sectoral response in six countries. London: Institute of Development Study.

25. International Institute for Applied Systems Analysis (IIASA). (2011): The Laxenburg Declaration on Population and Sustainable Development, Statement of a Global Expert Panel, octobre 2011, Laxenburg. Nations unies (2011), World Population Prospects: The 2010 Revision, Division de la population du Département des affaires économiques et sociales du Secrétariat des Nations unies, New York.

26. Marechal. (1963) : Essai sur le principe de population par Malthus Thomas Robert (Vol. I). (J.-P. MARECHAL, Éd., \& P. e. PREVOST, Trad.) Paris, France: GF-Flammarion.

27. Metahan Traore. (2010) : La croissance de la population et le problème alimentaire en Afrique, Mémoire de DESS Démographie, p.169. 
28. OCDE (2013) : Peuplement, marché et sécurité alimentaire [En ligne]. Cahiers de l'Afrique de l'Ouest, 208p. Disponible sur : https://read.oecd-ilibrary.org/agriculture-and-food/peuplementmarche-et-securite-alimentaire 9789264187412-fr (consulté le 20/02/2017) DOI : http://dx.doi.org/10.1787/9789264187412-fr DOI : 10.1787/9789264187412-fr

29. OCDE, (2014) : Rapport sur le cadre de l'OCDE pour une croissance inclusive [En ligne], Paris, OCDE, 68p. Disponible sur : https://www.oecd.org/fr/rcm/IG_MCM_FRENCH.pdf (consulté le $05 / 08 / 2017$ ).

30. Rossel. (2008): The impact of climatic shocks on child nutrition in Peru. Oxford: Young Lives - Oxford University.

31. Theil, H. (1963) : Essai sur le principe de population par Mathus. (B. Médiations, Éd.) Chicoutimi au Québec, Canada: Gonthier, page 12.

32. UNFPA. (2011b) : «Population Matters for Sustainable Development ", document d'analyse établi pour la consultation interorganisations sur la population et le développement durable (Interagency Consultation on Population and Sustainable Development), 23 juin 2011, UNFPA, New York.

33. Walter John .S. \& Schofield. (1989): Famine, disease and the social order in early modern society. (R. S. John Walter, Éd.) Cambridge: Cambridge University Press. 